\title{
What Is Collective Intelligence?
}

\section{I.I The Need for New Types of Collective Problem Solving}

In the new era of digital communication, collective problem solving is increasingly important. With the Internet and digitalization of information, large groups can now solve problems together in completely different ways than are possible in offline settings (Lévy, 1999). These novel online technologies and practices challenge our conceptions of individualized human problem solving in various domains, including art, science, industry, business, education, technology, software design, and medicine. It is urgent that we rethink our understanding of intelligence in a profound way. Among scholars, collective intelligence (CI) is increasingly used as a broad, multidisciplinary term to describe new types of collective problem solving. This notion of intelligence is not about individual ability or computer algorithms; rather, it describes how collectives of people, both small and very large groups, solve problems. This book intends to give an overview of some of the most important basic problem-solving mechanisms that comprise CI.

Throughout our evolution, our most extraordinary ability as humans is, without doubt, our ability to collaborate with each other. Our story is very much about how we gradually learned to solve problems together in increasingly larger groups. First, we started living in caves solving issues in small numbers, from there we formed villages, and, with time, the villages grew into kingdoms and nations. Today, many of us spend most of our time in a global online setting. In this new setting of billions of people, fresh ways of solving problems in large distributed groups are constantly being invented in a wide range of sectors. Open online innovation and citizen science are but a few examples of projects that center on open invitations, allowing anyone to join. In addition, various platforms and projects promote open online knowledge sharing, including the sharing of both knowledge products (e.g. online videos, Wikipedia) and knowledge 
construction processes (e.g. argument mapping). There is also a growing awareness that complex wicked problems, like climate change or COVIDI9, require innovative problem-solving approaches that build on the combined scientific and political efforts of individuals and groups all over the globe.

The increasingly popular concept of CI attempts to encompass this development across various scientific fields. Concerning group size, studies of CI cover anything from small group cooperation in teams in the offline setting to large group cooperation in distributed online settings (Salminen, 20I2). While some CI researchers still primarily examine the Internet and development of a broad macro level (Heylighen, 20 I7; Lévy, 20 Io), others focus on collaboration in small groups (Woolley, Aggarwal, \& Malone, 20I5; Woolley, Chabris, Pentland, Hashmi, \& Malone, 20ıо).

However, the invention of the Internet undoubtedly renewed interest in CI. Pierre Lévy coined the modern version of CI in 1994 with the book Collective intelligence: Mankind's emerging world in cyberspace. Inspired by the recent invention of the Internet, Lévy (1999) defines collective intelligence as a new universally distributed intelligence that constantly improves and coordinates itself in real time. For the first time in human history, the Internet made it possible for members of a decentralized community to interact with each other within the same virtual universe of knowledge. This made possible a new knowledge-producing culture that built on rapid and open exchange of data and ideas. Lévy predicted that this would lead to a fundamental change in how we think about ourselves. Knowledge will no longer be about established facts, but rather the essential part of an ongoing knowledge construction project that includes all humans. The fundamental premise is that nobody knows everything, everyone knows something, and all knowledge resides in humanity. Inspired by Verdansky's notion of "noosphere," Lévy predicts the emergence of a new collective intelligence at a global level (Lévy, 1999).

Since the World Wide Web was created in 1990, it has grown enormously from under 40 million users in 1995 to about $\mathrm{I} .5$ billion in 2009 (Castells, 2010). In 2020, an estimated 4.5 billion people are active Internet users, encompassing $59 \%$ of the global population (source: statista.com). The Internet makes it possible for most people on the earth to interact, create, and exchange information in new ways that extend previous space and time limitations (Castells, 2010). It builds on the instant storage and transmission of information with no loss. The speed of message transmission removes the problem of time delay and transport 
time. In principle, the outreach is global to all people who have access to the Internet. This permits flexible and easy communication between persons who are located in very different places (Brabham, 2013: I2-I3).

These capabilities make it possible to scale up activities and increase human collective capability in a range of different ways. As a result, people share information and communicate with each other in a huge range of online environments. During the last decade, participatory technologies, originally coined by Tim O'Reilly as Web 2.0 (Alexander, 2006), have connected a large amount of people and become increasingly important. As the first generation of web software in the I990s provided easy access to a vast amount of information, it was still technically difficult to publish information and produce web pages. The major change came with the second generation of Internet technologies, which made it easy for anyone to publish information and communicate with others. The Internet opened up a range of horizontal communication networks within social media, multiplayer online games and fan discussion communities. While the traditional mass media (television, radio, newspapers) had unidirectional links, the architecture in the networked information environment has multidirectional connections among all nodes (Benkler, 2006).

These networks are built around peoples' initiatives, interests, and desires and are used to share all kinds of digital information such as texts, photos, and videos. In social media, individuals constantly produce short texts (e.g. Twitter), images (e.g. Facebook), or videos (e.g. YouTube). These short messages are part of an ongoing online social dialogue, and they are viewed by others immediately afterwards. Online cultural expressions and personal experiences have become a fundamental part of our daily life in the last decade (Castells, 2010). In addition, these new networks integrate local and global media and transcend traditional space limitations.

A fundamental premise behind this development is the radical reduction of the cost of becoming a speaker. Because the cost is so low and it takes very short time to reach others over the Internet, more people can find each other and create something together. Before the age of the Internet, there were only a few people who published their knowledge and opinions to a wider audience, and the publishing channels were usually under editorial control. Now anyone that can afford a digital device (like a cell phone or laptop) can access the Internet and produce and publish digital information. One consequence is that the traditional expert model of knowledge production, which has been taken for granted for centuries, is now being challenged. Increasingly, experts today not only compete for 
attention with each other, but with a large number of influencers and other amateurs who create, publish, and share their own content. In this networked information economy, knowledge production is much more broadly distributed in society.

Some of these large, loosely organized groups of people have also been surprisingly successful in building new knowledge products of societal value. The rise of effective, large-scale cooperative efforts like Wikipedia, which build on peer production of information, knowledge, and culture, was considered to be the most radical new innovation in the network society (Benkler, 2006). In the early 2000s, these new global online communities gave promise of a bright new future which would bring people from all over the world together. This development spurred a new era for CI research. A decade ago, the research report "Harnessing Crowds: Mapping the Genome of Collective Intelligence", Malone, Laubacher, and Dellarocas (2009) helped form a preliminary overview of what could be regarded as a new research field. Inspired by global online networks and communities like Wikipedia, the report proposes a relatively detailed typology, specific "building blocks," that can guide the design of $\mathrm{CI}$ communities. The researchers also claim that CI has existed throughout history. Therefore, the basic mechanisms are not new, but the main difference is that the Internet has created a new type of web-enabled CI that have resulted in new practices in fields like business and science. However, the link between our present and previous history is not clarified, and leaves the question open on how these new online practices are similar or different from previous ways of solving problems.

Today, CI has become a multidisciplinary notion within a range of different areas. The concept is used within disciplines such as psychology (Woolley \& Aggarwal, 2017), political science (Landemore, 2013), business (Täuscher, 20I7), complexity sciences (Heylighen, 20I7; Stefanelli et al., 2019), biology (Bonabeau, 2009; Ioannou, 2017; Vercammen \& Burgman, 2019), computer sciences and semantics (Alag, 2009; Lévy, 20ıо; Lollini, Farley, \& Levy, 2019), and social media research (Schoder, Gloor, \& Metaxas, 2013). The recommended list of topics at the annual conference on $\mathrm{CI}$ in 2020 illustrated the rich variety of topics: human computation, social computing, crowdsourcing, wisdom of crowds (e.g. prediction markets), group cognition, collective decision-making and problem solving, participatory and deliberative democracy, animal collective behavior, organizational design, public policy design, ethics of collective intelligence, computational models of group search and optimization, emergence and evolution of intelligence in biological systems, new 
technologies for making groups smarter, collective creativity and innovation, citizen engagement and participation, citizen science, artificial intelligence and collaboration, open source intelligence, collective computation, swarming, voting mechanism design, and collective forecasting (Intelligence, 2020). This overview shows that many different disciplines address separate aspects of collective intelligence. CI encompasses a wide range of practices that move beyond the individual level to include groups of peoples of various sizes who use different types of technology (Mulgan, 2014, 2018).

However, since CI is a relatively new academic concept, there are only a couple of books that aim to provide a broad overview of the concept, the field, and the different CI practices (Malone, 2018; Malone \& Bernstein, 20I5; Mulgan, 2018), including a few review articles (Peters \& Heraud, 2015; Suran, Pattanaik, \& Draheim, 2020). Although these publications represent important steps toward unifying the field, they also show how hard it is to summarize the field, primarily because of the lack of shared concepts. Separate disciplines use their own terminology within their own silo and there are few multidisciplinary studies. Although each discipline provides useful research, there is still no general framework that all disciplines can draw on which can provide a shared understanding of the basic mechanisms behind CI (Mulgan, 2018: 229-230).

According to Mulgan (2018: 229-230), the CI literature ranges from the limitlessly broad to the highly specific. The narrow variants describe collaboration in small groups, while the broader variants describe the whole of human civilization and culture (Mulgan, 20I8: I). For example, there is disagreement on whether collaboration in teams or smaller groups in an offline setting should be included in a definition of collective intelligence. Aulinger and Miller (20I4) claim some definitions of CI imply that almost any collective action can be labeled as "collective intelligence." With this lack of precision, the concept may end up meaning nothing. They suggest the exclusion of small groups or team intelligence from a definition of CI. Instead, they propose that $\mathrm{CI}$ should focus on how individuals follow identical rules. This emphasis on a narrow variant of CI illustrates the conceptual struggle in this multidisciplinary field. Here, the basic question is whether CI studies of small group collaboration have anything in common with collective work in large global online communities. If this is the case, this connection needs to be further explained within a shared conceptual framework.

Because CI is a new research area, a range of other terms are obviously also used to describe the same or similar practices. One example is crowdsourcing (Brabham, 2013) or swarm intelligence (Corne, Reynolds, \& Bonabeau, 20I2). CI is also used to discuss 
nonhuman intelligence in some research areas, both animal intelligence and machine intelligence. In one review, Salminen (2012) found that only $25 \%$ of the papers on CI discuss human intelligence. A majority of the papers discuss collective behavior of cognitively simple agents such as insects, robots, and simulation algorithms. One area addresses new programming techniques used to analyze large amounts of quantitative data, which people leave behind when they use the Internet (e.g. Alag, 2009).

Although the focus of human CI research varies substantially, the shared assumption is that intelligence builds on some type of collective interaction or problem solving. It is something more than a psychological ability residing inside the head of an individual. For example, Jenkins (2009) challenges the view of intelligence as an attribute of individuals, and instead describes $\mathrm{CI}$ as being a new type of intelligence distributed across an extended technological and sociocultural online environment. In line with perspectives from distributed cognition, CI practices "offload" information into the environment.

\section{I.2 Theoretical Perspectives on CI}

As a scientific field, CI is still largely undeveloped and untheorized. There are relatively small research communities within areas such as computer science, psychology, economics, and biology. Some research studies also examine the interplay between human collective behavior and machine learning, but it is still not clear how CI differs from machine learning. There are few usable theories and a lack of analysis of CI at a large scale in organizations, cities, nations, and networks (Mulgan, 2014, 2018). Typologies are practice-centered, often aiming to categorize and synthesize different online CI practices without any use of a dedicated theoretical framework (Malone et al., 2009; Suran et al., 2020).

Despite the lack of coherence, the scientific community has still identified some important mechanisms across different disciplines. First, at a micro level, empirical studies have identified a general group intelligence factor that explains problem solving in small groups. Second, many largescale studies of collective work are explained through different selforganization mechanisms. Third, a vast number of CI studies, covering both a micro and macro level, address the role of informational diversity or cognitive diversity in different ways.

I. A general group intelligence factor

2. Self-organization

3. The role of diversity 


\section{I.2.I A General Group Intelligence Factor}

Historically, the invention of the intelligence test establishes the intelligence concept. In 1905, Alfred Binet designed the first version of this test. It identified French schoolchildren with learning disabilities who needed more support than other children (Binet, Simon, \& Kite, I9I6). At the same time, Charles Spearman (1904) developed the theory of general intelligence (or " $\mathrm{g}$ ") that proposed that a large part of a person's intelligence was built on a general problem-solving ability. It would persist for many years before more complex definitions of intelligence were accepted (Piaget, 1952). In recent time, there have also been attempts to extend the notion of intelligence beyond its focus on human cognition. For example, Howard Gardner (1983) described the existence of seven different types of intelligence in his book Frames of Mind: The Theory of Multiple Intelligences. Three types covered cognitive abilities (linguistic intelligence, logical-mathematical intelligence, and spatial intelligence), and the four others, musical intelligence, bodily-kinesthetic intelligence, interpersonal intelligence, and intrapersonal intelligence, were new types of intelligence. Intrapersonal intelligence focuses on the capacity to have knowledge about oneself and control personal emotions, while socially orientated interpersonal intelligence describes the ability to understand and collaborate with other people. Still, human intelligence today is primarily connected to cognitive abilities and skills.

In contrast, CI research by Woolley et al. (2010) have found evidence of a general group intelligence factor, labeled the "c factor," in different types of group work. This has even led to the development of a group intelligence test, which is different from the cognitive tasks that are typical in standardized individual intelligence tests. The test tasks cover four different dimensions in authentic settings. The first task is about generating something new, like brainstorming various uses for a brick. The second category involves the selection of a pre-specified alternative, making groups solve visual puzzles from a standardized test called Raven's Matrices. The third dimension includes negotiating tasks, challenging the group to pretend they live together and have to plan a shopping trip. The fourth dimension is about executing tasks, and letting the group type a long text passage through synchronous online writing. In addition, other tasks involve word-completion problems, spatial puzzles, and estimation problems (Malone, 20 I 8: 3I).

In the original study, 152 groups of two to five members were set to solve a wide range of different tasks. Factor analysis of team scores 
identified one factor accounting for 44 percent of the variance, while the second factor only explained 20 percent of the scores. Here, collective intelligence is the inference one draws when the ability of a group to perform one task is correlated with that group's ability to perform a wide range of other tasks. The first factor with significant explanatory power is interpreted as a property of the group itself, not just the individuals in it. Nor was this factor correlated with the average or maximum individual intelligence of group members (Woolley et al., 2010). Other follow-up studies have shown similar results in other settings across different languages, cultures, and activities (Malone, 2018: 32-42; Woolley et al., $2015)$. For example, in high-performing teams playing online video games, collective intelligence scores were significant predictors of their performance in the game (Kim et al., 2017). The "c factor" has also predicted performance for other more complex tasks such as playing checkers against a computer or solving architectural design problems. In addition, the highly collectively intelligent teams exhibited steady improvement in performance across the series of tests, indicating that these groups also learn faster (Malone, 2018: 32-42; Woolley et al., 2015).

According to Malone (20I 8: 4I), the combination of all these studies indicate that human groups have a kind of collective intelligence that is directly analogous to what is measured by individual intelligence tests. $\mathrm{He}$ highlights the distinction between (I) specialized intelligence and (2) general intelligence in individual intelligence tests (Malone, 2018: 24). First, specialized intelligence refers to the ability to achieve specific goals effectively in a given environment. The equivalent of this type at a group level will then be "group effectiveness." However, intelligence tests have been designed to predict your general intelligence or your ability to do a wide range of other tasks beyond those in the test. People who have much of this general intelligence are better at adapting to new environments and learn more quickly. Likewise, general collective intelligence refers to the group's ability to adapt to new environments and perform well on a wide range of different group tasks (Malone, 201 8: 24-25, 4I).

Although some researchers claim there is insufficient support for the existence of a collective intelligence construct (e.g. Bates \& Gupta, 20I7; Credé \& Howardson, 20I7; Woolley, Kim, \& Malone, 20I8), there is increased interest in the more general problem-solving abilities in groups in both offline and online settings. However, we still know little about which group processes or group qualities influence the "c factor." There are affiliated concepts such as group cognition and group mind. Within sociology, both Durkheim's concept of collective consciousness and 
Gabriel Tarde's notion of group mind move beyond the individual self in their examination of societal beliefs in larger groups. In psychology, new theories of learning also highlight the qualities of group discourse and joint meaning making to a greater degree (Sawyer, 2006; Stahl, 2006). Knowledge does not reside inside the heads of individuals, but in the practice itself (Flick, I998; Gergen, I985). Likewise, this book analyzes CI as a group phenomenon.

\section{I.2.2 Self-Organization}

Another strand of CI research examines different types of self-organization. The first type of self-organization is at a macro level, describing the Internet as a self-organizing super-intelligence that unites all human intelligence into a worldwide network of information and communication. For example, Heylighen (20 I I) uses the metaphor of a global brain to describe the Internet as an intelligent, organism-like system, a brain of brains. CI emerges from the collective interactions between humans and machine in a global online communication network. This global brain is immensely complex and self-organizing without any centralized control, and emerges as an adaptive complex system. In an interview (Lollini et al., 2019), Levy claims this type of self-organization can best be described as stigmergic communication. Throughout our human history, improvements in CI has followed from inventions that augmented the power of human language. The invention of writing created a new collective memory that was further developed with the invention of the printing press. Moreover, the invention of the Internet completely removes the constraints of physical space and memory when knowledge becomes accessible from anywhere in the world. This is not only communication from many to many, but also a new way of connecting knowledge when it is stored in an online setting. The stigmergic element refers to the intermediary of a common shared environment that everyone uses. Almost the entirety of humanity can add knowledge to this shared memory, which anyone can access. In addition, every new trace of action on the Internet will continuously change the relationship between the stored digitized data. In this sense, everybody contributes to the transformation of the common memory at the same time. Although CI is facing huge challenges today, Levy proposes that the way forward is to design practices that can promote reflective communication between people in the online setting (Lollini et al., 2019).

The second type of self-organization describes the emergence of global online communities. One example is Wikipedia, which has more than six 
million articles in the English version alone (Rijshouwer, 2019). Another example is the development of open source software where many individuals contribute at different points of time (Raymond, I999). Mulgan (2018: 76) also describes how stigmergy is important in self-organizing systems like Wikipedia, or among open source software development programmers who pass around tasks in the form of challenges until they find a volunteer. Human stigmergic problem solving is an important part of the analysis in this book (see Chapter 6).

Third, self-organization can build on market mechanisms, like the "invisible hand" that self-regulates the market economy by letting everyone pursue their own interests (Hayek, 2013). Widely dispersed markets use price signals efficiently to coordinate large-scale activities. Markets can adjust prices with little horizontal communication between the participants, but they are limited to the binary decision of whether or not to buy something (Mulgan, 2018: III, II5). In CI research, this type of selforganization has been examined in studies of prediction markets (Buckley \& O'Brien, 2017; Malone, 2018) which is also a topic addressed in this book (see Section 6.3).

A fourth type of self-organization studies swarm problem solving in animals. Peters and Heraud (2015) claim biological studies of "swarm intelligence" is one of six major areas within CI. It refers to the collective behavior of social insects and flocking behavior (Mulgan, 2018: 232). For example, Sumpter (2010) claims human collective behavior can be explained through self-organization and different behavioral algorithms. These principles, such as positive feedback, response thresholds, and independent decision-making, are also present in different animal groups and can inform our understanding of human societies. However, Willcox, Rosenberg, and Domnauer (2020), claim there is no good theory that explains how human swarms operate. Few studies examine large-scale human collective work in the offline setting. This area of investigation is labeled as human swarm problem solving in this book (see Chapter 4).

\section{I.2.3 The Role of Diversity}

In general, CI expects that new technologies will make groups better at solving problems than ever before (Malone, 2018). The predominant strategy is to scale up the size of the group and hope this can create more diversity benefits. A prominent example is the book The Wisdom of Crowds by Surowiecki (2005), which describes four qualities that make a crowd intelligent. First, the group should be diverse, so different individuals can 
supplement each other with different pieces of information. Second, the group needs to be decentralized, without anyone directing the answers from the center. Third, individual opinions need to be aggregated into a collective opinion. Aggregation typically depends on numerical contributions and statistical methods. Fourth, the individuals in the crowd should act independently of each other.

In a historical perspective, it was the British scientist Francis Galton who in 1906 first described the "Wisdom of the Crowd"-effect from a scientific perspective. He visited West of England Fat Stock and Poultry Exhibition, which had organized a contest where anyone could guess the weight of an ox. Eight hundred individuals made guesses, but none had the right answer. Galton decided to average all the guesses, and surprisingly discovered that the estimate of $\mathrm{I}, \mathrm{I} 98$ pounds was only 0.8 percent different from the correct answer of I,207 pounds (Galton, 1907). It was the first scientific paper suggesting that a large group could be much more accurate than individuals.

Today, the diversity prediction theorem, developed by Hong and Page (2004) represents perhaps the most important theoretical explanation of CI with its emphasis on diversity. The mathematical theorem explains the relationship between collective accuracy and the diversity of predictions based on expected errors. The theorem can be written as the following mathematical proof:

The Crowd's square Error = Mean square error of individuals Predictive Diversity (Hong \& Page, 2004).

The theorem states that the error of a crowd equals the average squared error minus the predictive diversity. First, the mean square error is the average of the individual squared errors. It includes the errors each individual has made as a distance from the correct or true value (Page, 20I4).

Second, the prediction diversity equals the average squared distance from the individual predictions to the average prediction. From a statistical perspective, this is the same as the variance or how widely spread the predictions are, but Page prefers to use diversity as a term to underline the importance of variations in the predictions. This is the crowd diversity dimension (Page, 2014).

Third, the crowd error is the squared error of the collective prediction. It equals the average squared error (crowd's prediction) minus the predictive diversity. The crowd square error must always be smaller or equal to the mean square error. Consequently, the prediction of a crowd must always be better than or equal to the average prediction of its members. Much better prediction requires a larger degree of diversity, while a crowd that is 
only as good as its average member will have no predictive diversity. The main point is that when the diversity in a group is large, the crowd error will be small. Therefore, wise crowds will rely on not only individual accuracy (mean individual error) but also crowd diversity (Page, 20I4: I332).

Consider the following example. Two persons predict the annual snowfall in their hometown. Both deviate from the correct answer by 4 inches, making the squared error equal to I6. If both guess 4 inches too many or too few, there is no predictive diversity. The crowd error will then be 16 , equal to the mean square error (Theorem score: $16=16-0$ ). However, if one person predicts 4 inches too many and the other person 4 inches too few, the crowd will provide the correct answer because the diversity of predictions cancel each other out (Theorem score: $\mathrm{O}=\mathrm{I} 6-\mathrm{I} 6$ ) (Page, 20I4).

In essence, this theorem points to cognitive or informational diversity being at the core of $\mathrm{CI}$, and this book will further explore this topic in the discussion of different types of collective problem solving.

\section{I.2.3.I The Many Wrongs Principle}

Furthermore, the "many wrongs principle" and "the many eyes" principle are two different principles that explain the benefits of diversity. In the "many wrongs principle," or "the generated framework" (Page, 20I4), predictions are modeled as the truth plus a disturbance. For example, when a group of persons individually estimate (predict) the height of a tree, each person will observe the height from a slightly different position on the ground. Because of these variations in vantage point, each individual observes the true height plus some error term. When these errors are made independent of each other, they will be diverse, and the aggregated crowd error will be small because the individual random errors cancel each other out (Page, 2014).

Answers that aim to be accurate must avoid systematic bias. For example, in guessing the weight of the ox at the county fair, Galton (1907) noticed that the individual judgements were less biased by passion and joking because contestants had to pay a small fee to compete. This prompted each competitor to do his best. The competition for prizes probably also motivated contestants to make independent judgements and not discuss their estimates with other contestants, thus reducing the possibility of systematic bias.

According to the "many wrongs principle," the crowd wisdom builds on the aggregation of individually independent guesses that have random or 
symmetrically distributed errors. When many people with no particular bias make an estimate, they will be equally likely to make errors on the high and low side of the correct answer. By averaging the answers, these errors cancel each other out because of the law of the large numbers. Under these circumstances, the larger the crowd, the more accurate the estimate. The limitation is the requirement of numerical contributions, which leaves out many other types of collective problem solving (Krause, Ruxton, \& Krause, 20I0; Malone, 2018; Page, 2014).

\section{I.2.3.2 The Many Eyes Principle}

The "many eyes principle," or the "interpretive signal framework" (Page, 20I4) provides an alternative explanatory framework by assuming that accurate collective predictions build on diverse mental models. Because people use different models, perspectives or heuristics when they solve a problem, they also make different mistakes. These techniques or strategies will vary depending on variations in life experiences, cultural background, and formal training (Page, 20I4). For example, if you estimate the temperature outdoors, you will use your tacit "personal knowledge" that builds on previous experiences of estimating the temperature. When a group does this task, they will operate with uniquely different mental models, and the aggregated average of the temperature will therefore often be very accurate.

When guessing the height of a tree, the "many eyes principle" does not only include individual differences in vantage point, or the distance to the tree, but it also involves the differences in the cognitive strategies individuals use to estimate the actual size of the tree. For instance, do you try to compare the height of the tree with other objects close by or do you know how tall such trees usually are? When individuals build their estimation on different heuristics, this increases the cognitive diversity and helps provide a more precise estimate at an aggregated level. In the "many eyes principle," individuals filter the world in their own unique way, and therefore they will observe different approximations of the same phenomenon (Page, 20I4).

According to this principle, the wisdom of the crowd in the ox contest is not about errors that cancel, but it is about the crowd providing a more complete explanation. At the county fair, a relatively large group of contestants was highly competent since they were butchers or farmers (Galton, 1907). According to the "many eyes principle," these individuals would still probably have used different heuristics when estimating the weight of the ox. One cognitive strategy could have been, "The ox is about ten times my size - I weigh 90 kilos - therefore the ox should be around 
900 kilos." Another strategy could have been, "Oxen at my farm usually weigh I, Ioo kilos, this ox seems to be a bit larger than average, so the weight should be around I,200 kilos." The contestants who were not butchers may also have contributed with important "bonus diversity" by using relevant heuristics that are significantly different from how butchers or farmers estimate the weight. Here, the threat is that many in the crowd use the same mental models, which then leads to less cognitive diversity and a more imprecise aggregate estimation.

This implies that the collective problem solving was relatively accurate even with diverse individual perspectives. According to the "many eyes principle," errors still cancel out, not because of randomized draws, but because individuals use distinctly different mental models that together provide a better "collective mental model." Because the world becomes more complex and harder to predict, an individual model is likely to produce a large error, resulting in a large mean error on the aggregated level. Then, according to the diversity prediction theorem, the only way to keep the crowd error small is to ensure that the predictive diversity is large (Page, 20I4).

Another implication of the "many eyes principle" is that it is risky to select team members based on their ability because they are then likely to be less diverse from each other. The well-known catchphrase that "diversity trumps ability" was originally inspired by a computational experiment by Hong and Page (2004), where the simulation results surprisingly showed that a diverse, randomly selected group of agents outperformed a group of the best agents. The reason this happened is because in large populations, the functional diversity of the group of individually bestperforming agents becomes very small. If you choose the two best problem solvers from a large set, they are more likely to use similar perspectives and heuristics. Under certain conditions, the model predicts that diversity trumps ability, implying that it is better to select team members based on diversity of heuristics than their individual ability. The best problem solvers tend to be more similar, and IQ test scores will therefore not necessarily be a good predictor of the team performance. The exception is simple problem solving, such as some types of physical labor, where the individual with the highest ability will also be the best team member (Page, 20I4).

The diversity prediction theorem covers collective problem solving at both at a micro group level (Page, 2017) and a macro group level (Page, 2008). It has inspired theoretical work within epistemic democracy (Anderson, 2006) and experimentation with new democratic models that can better tap the "wisdom of citizen diversity" (Landemore, 2013). 
Although some question the relevance of using mathematical models to describe cognitive diversity, the diversity prediction theorem still constitutes an important theoretical premise for CI research. Cognitive diversity assumes that better solutions build on a broader set of perspectives that look at different parts of the problem (Page, 2018). Similarly, this book describes distinctly different types of collective problem solving that aim to provide a more comprehensive understanding of CI.

\section{I.3 Top Solvers in Online Innovation Teams}

In addition to reviewing relevant theory and CI practices, this book will also examine new data from online innovation contests. These contests are an important part of CI research. Page (2014) mentions these contests as an interesting example of a new type of cognitive diversity that enables thousands of problem solvers to participate in complex problem solving and thus increase the likelihood of producing an optimal solution. Today, specialized online innovation intermediaries often host these online innovation contests.

In recent years, it has become more common to enable teams to solve challenges instead of having a large number of individuals working separately from each other. Multidisciplinary teams can work on challenges that are more complex by moving beyond simple aggregation, towards combining and synthesizing ideas.

Several chapters in this book will include data on how top solvers experience participation in such online contests, including both small teams and larger groups. The data consist of selected excerpts from 80 interviews published on the IdeaConnection website, one of the most prominent online innovation intermediaries. All the solvers in the corpus have won a contest, so they are not representative of the large member database, which includes many who have not won any contests. These solver stories provide detailed, illustrative descriptions of the different types of collective problem solving, especially collaborative problem solving.

The contest format at IdeaConnection has several design features that aim to utilize cognitive diversity. The innovation teams will typically be both multidisciplinary and multicultural, and a successful solution will usually depend on this diversity. In this team contest format, individuals are invited to participate in teams comprising four to five persons and a facilitator. Only a few teams will compete for the prize, which increases the chance of each team winning, compared with the contests in which anyone can participate. At the same time, the diversity of proposed solutions 
increases because the competing teams develop solutions independently of each other. These teams are also interesting because they illustrate complex problem solving in an online setting. They point towards a future where online CI is likely to become more important in collaborative problem solving. While a significant amount of CI research today consists of experimental studies, these teams represents an interesting supplement in their descriptions of how collective problem solving unfolds itself in "natural" online settings.

\section{I.4 A Cultural-Historical Perspective on CI}

According to Mulgan (2018: 2), CI needs to address the big question today; how can societies and governing systems solve complex problems, or how do collective problems find collective solutions? Until now a lot of the CI research has addressed relatively simple one-dimensional problems, while some of the most pressing tasks today reveal conflicting interests and less clarity about what answers are right, which only time can resolve (Mulgan, 20I 8: 26).

Machine intelligence and artificial intelligence (AI) is also another important area within CI research (Peters \& Heraud, 2015). However, this book will highlight this type of $\mathrm{CI}$ as something different, being primarily a human-to-human intelligence. CI mobilizes human intelligence at scale, often linked through the Internet, and includes new ways of organizing knowledge production and solving problems, as in crowdsourcing (Mulgan, 20I8: I6). Although machine intelligence and $\mathrm{CI}$ are often closely connected with each other, $\mathrm{CI}$ is assumed to build on a different logic. According to Mulgan (2018: 237), CI is the capacity of groups to make good decisions through a combination of human and machine capabilities. Our lives will in the future obviously become even more interwoven with machine intelligence that both challenges and amplifies us, but human collectives and human intelligence must still be at the center (Mulgan, 2018: 6, 235).

As such, this will not be a book about machine learning or AI. Even without computer science, the CI field is very broad. It covers both small groups and large groups, and offline and online settings. One might even ask whether there exists any general mechanisms across the multitudes of settings and group sizes. Until now, most studies of CI describe new online practices. Although a range of multidisciplinary models and definitions have been introduced (e.g. Suran et al., 2020), none have, to my knowledge, examined CI within a historical perspective. One reason may be that 
the short format of a research article limits the possibility for detailed historical examination.

This book will address the lack of research in this area by analyzing how CI has evolved in a historical perspective. By including this perspective, one avoids perspectives that rely too heavily on technological innovation in itself, with the risk of ending up in technological determinism. Still, this book assumes that it is not enough to adopt a historical understanding, since major technological innovations are changing the way we solve problems. CI should align with both former and recent historical development.

From a Vygotskian perspective, the explanation of any human phenomenon, including CI, should consider both biological and cultural-historical perspectives. A scientific study should not only focus on CI as an improved product of what groups can achieve, but also investigate the processes by which a phenomenon emerges and how it originated. The emphasis is on human cognition in growth or transition, where different forces of development follow their own logic. Both natural and cultural lines of development interact with one another, but they are not necessarily united. It is only through analytical abstraction that we can separate one set of processes from others. A complete analysis of human psychological processes should still aim to integrate these perspectives and their corresponding explanatory principles (Wertsch, I985: $17,4 \mathrm{I}-42$ ).

Inspired by this methodology, CI is analyzed as a phenomenon comprising three types of collective problem solving: collaborative problem solving, swarm problem solving and stigmergic problem solving. These problem-solving types are not final or complete in any way. However, if analyzed in combination, they provide a set of explanatory principles that contributes to a more complex understanding of CI. The different types of collective problem solving include a range of different practices at different scales and levels, including both group work in a face-to-face offline setting at a micro level and large-scale collective work in an online setting at a macro level. In this sense, the book aims to contribute in establishing a full-fledged discipline of collective intelligence (Mulgan, 20 18: 4).

This book also examines this issue by describing the origins of the three different types of collective problem-solving types. Although online practices are new, our societies accumulate knowledge and develop according to specific historical mechanisms. Different types of collective problem solving evolved gradually into more complexity in human history. This is perhaps most evident in the chapters about the origins of human swarm problem solving (see Chapter 5) and human stigmergic problem solving 
(see Chapter 7). The examination of the history of CI can also disclose core mechanisms in collective problem solving that are still relevant for online CI practices today.

Furthermore, a cultural-historical perspective avoids a reductionist approach that provides an overly simple description of CI. The goal with this book is to shed light on how CI practices today can contribute to the development of a better society and not just improved progress within a few narrow problem areas (Mulgan, 20I 8: 223). Hence, Chapter I 3 analyzes the COVID-I9 pandemic from a CI perspective. According to Mulgan (20I8: 6), CI is in many ways humanity's grandest challenge, since we need to develop our ways of thinking and acting together if we are to solve the other grand challenges of climate, health, prosperity or war.

\section{I.5 The Methodological Steps}

The methodology in this book follows four steps, explained below in more detail.

\section{I.5.I Step I: Review Current CI Practices}

In the first phase of this research process, I undertook an extensive search of CI literature to obtain an overview of the most common practices and characterizations of the phenomenon. This phase dates back to the reading of a report in 20 I (Malone et al., 2009). Over the following years, several new scholars and stakeholders introduced new models covering a range of fields and sectors; however, these models typically concern a relatively small part of the multidisciplinary field. Chapters I -3 comprise a summary of these readings on CI. They constitute the main review of CI literature, dividing the field into crowdsourcing and open online knowledge sharing as two major areas. Within these areas, a few important CI practices have been selected to provide a more detailed account. This approach coincides with Mulgan (201 8: 236), who suggests that an emerging discipline should be descriptive and analytic, observing collective intelligence "in the wild" in finding the most successful CI practices.

Chapter 2 describes crowdsourcing, a process whereby problems are sent outside an organization to a large group of people - a crowd - who can help provide solutions (Surowiecki, 2005). Online citizen science and online innovation contests are of particular interest because of their societal value. Within innovation, the two selected examples are from IdeaConnection and Climate CoLab, two innovation intermediaries who 
host different types of online innovation contests. One of these contests, the IdeaRally, represents an interesting new crowdsourcing method that allows hundreds of experts to participate in a one-week long intensive idea building process. In online citizen science, Zooniverse (e.g. Galaxy Zoo) and Foldit are selected as two prominent, but contrasting examples. The online protein folding game Foldit stands out as a particularly successful project that show what amateur gamers can achieve. The game design combines human visual skills with computer power in solving proteinstructure prediction problems by constructing three-dimensional structures. Most successful solutions are team performances or achievements made by the entire Foldit gaming community. All the examples in this chapter illustrate successful case stories, and the detailed analysis identifies basic problem-solving mechanisms in crowdsourcing.

Another important area in CI is open online knowledge sharing (see Chapter 3). Open sharing is becoming more important in all major sectors in society, including science, politics, education and innovation. This sharing includes both the domain of expert-produced scientific knowledge and massive amounts of citizen-produced practical knowledge. Because of lower publishing costs, Open Access has become the new dominant trend that makes research accessible to everyone. Increased production of open textbooks gives a more readable access to scientific knowledge and reaches a much wider audience. In addition, scientific knowledge construction processes are becoming transparent. This includes the establishment of many more open digital databases that allow anyone both to make their own contributions and get free access to all the data (e.g. citizen science project like eBird). There is also experimentation with making knowledge construction processes more open, both within scientific discourse (e.g. Polymath project) and the development of encyclopedic knowledge (e.g. Wikipedia). In addition, the recent decade has seen an enormous increase in amateur-produced practical knowledge, not only texts, but an abundance of images and videos. Enthusiasts share their skills and passions concerning any activity that might be of interest to other like-minded persons. It also includes the sharing of political opinions, for example with new digital technologies like argument mapping. Even some companies in the business sector have begun sharing more of its corporate knowledge.

These CI practices address aspects of what Peters and Heraud (2015) label as social innovation, new social practices that aim to strengthen civil society by improving working conditions, education, community development or health. This approach assumes that complex social problems require the involvement of engaged citizens. Citizens are capable of 
both co-evaluating and co-creating public goods and services that can reform the public sector (Peters \& Heraud, 2015).

My perusal of the literature suggests that both crowdsourcing and open online knowledge sharing are central to CI, areas often highlighted at conferences, in research papers or in books on the subject. However, this does not imply that the CI examples are mainstream today. One example is argument mapping, an interesting practice that is not widely used. Online innovation contests and online citizen science (Chapter 2) are new, but still relatively peripheral practices.

\section{I.5.2 Step 2: Identifying CI as Three Types of Collective Problem Solving}

Collective problem solving is the core term in this book about CI. The term covers a range of different practices across different group sizes and periods, while retaining a common emphasis on aspects of problem solving. The term differs slightly from what is typical among other CI researchers, who often underline communication, coordination or other system characteristics (e.g. Suran et al., 2020). The main advantage with using problem solving as a term is that it intends to cover the "complete" intelligent process. My readings of biologically orientated CI research inspired me to distinguish between three types of collective problem solving: I. collaborative, 2. human swarm and 3. human stigmergic. Research on both swarm behavior and stigmergy is relevant for a large range of collective practices today. Subsequently, I searched for additional research studies to enrich the descriptions of the particularities of these subtypes (Chapters 4, 6, and 8), even when these studies did not explicitly mention CI. The goal in the first part of the book is to give a detailed description of the basic mechanisms that characterize these three types of collective problem solving.

Chapter 4 discusses human swarm problem solving as a distinct subtype of CI with biological antecedents in nest siting among honeybees and flocking behavior. Building on recent biological research, this chapter discusses five mechanisms that are also relevant for human swarm problem solving. These mechanisms are decision threshold methods, averaging, large gatherings, heterogeneous social interaction and environmental sensing. Studies of collective animal behavior show that they often make decisions that build on statistical rules (e.g. averaging, threshold responses). Even when in a group, individuals will often seek and assess information independently of others with the intention of optimizing decisions through the "many wrongs principle" or the "many eyes 
principle." Similarly, human "wisdom of the crowd" studies examine similar statistical rules and principles like the importance of making independent contributions. However, while early research on the wisdom of crowds addressed the importance of independent contributions, newer studies also examine the possible positive influence of dependent contributions. The increasing variety of crowdsourcing studies are in this chapter explained within the framework of different swarm mechanisms. In the summary, four basic characteristics of human swarm problem solving are highlighted: predefined problems, pre-specified problemsolving procedures, rapid time-limited problem solving, and individual learning.

Chapter 6 presents human stigmergic problem solving as a distinct "solution-centered" subtype of CI with biological antecedents in the trail-laying and nest-building of ants. Stigmergy describe how many individual agents are able to coordinate collective action only by leaving information in a shared environment. In this type of collective problem solving, a version of a solution will already exist, either partially or completely. The problem-solving process will, therefore, be a response that changes the existing version of a solution by rating it, in the case of, for example, an online video; re-estimating it through a prediction market; adapting it like an open textbook or completing it like a Wikipedia article. In human qualitative stigmergy, a preliminary part of a solution will be stored in the system or medium, and individuals will then respond to the unfinishedness of the solution in different ways. If many versions of a solutions already exist, human quantitative stigmergy can also be used to rate the most optimal solutions. In the online setting, solutions will be continuously compared with each other. These stored solutions can solve many different problems at various points of time.

Chapter 8 proposes collaborative problem solving as one of three distinct types of CI. Collaborative problem solving covers a wide range of disciplines and contexts, but this chapter primarily draws on studies that have explicitly used CI as a scholarly concept. The most important finding is the identification of a general group performance ability on a wide variety of tasks. This group performance is analyzed in relation to four dimensions that promote successful collaborative problem solving. First, "working well with others" is not only analyzed as an individual ability, but as a quality that emerges through the qualities of a symmetrical collaborative relationship. Second, "cognitive diversity" describes diverse repertoires in groups, also including multidisciplinary and multicultural diversity. Third, "equal participation" emphasizes that everyone in the group should 
be allowed to bring in their perspective, and group discussions need to be open-minded. Fourth, "joint coordination" is important in setting goals together, dividing tasks and choosing relevant problem-solving strategies. Solver experiences from online innovation teams exemplify how this type of CI can move forward in a highly relevant authentic online setting.

\subsubsection{Step 3: A Historical Analysis of the Problem-Solving Types}

The third step is a cultural-historical analysis of each of the three types of collective problem solving (Chapters 5, 7, and 9). It is assumed that all problem-solving types build on mechanisms that humans already use. Hence, both the historical and phylogenetical origins of these three types of collective problem solving and their inner contradictions are examined. In addition, this brief analysis explains how the different problem-solving types evolved in complexity until our present day. CI is analyzed as a practice that has evolved over time as humans have learned how to use increasingly advanced tools. Sources include various previous books and articles that describe the relevant historical practices.

Chapter 5 argues that the origins of human swarm problem solving can be traced back to group hunting, which required rapid problem solving during the hunt, but also planning activities. Collective actions build on synchronization in the sense that every contribution from individual hunters mattered. Another milestone was the emergence of premodern trade, which enabled human groups to utilize informational diversity from nonkin and even strangers. Knowledge was shared in new ways through large gatherings and trade networks. The third major achievement was the establishment of the first democracy in ancient Athens with institutions such as the Assembly of the People, the Council of 500 and the People's Court. These institutions enabled a large number of individuals to engage in rapid problem solving in a formalized manner. Individuals from all over the Athenian territory met in the city to solve societal problems. These historical examples show that human swarm problem solving is also a story about our ability to solve problems in increasingly larger groups.

Chapter 7 trace the origin of human stigmergic problem solving back to the invention of writing. Knowledge could now be stored, reused and made accessible to others. A human collective memory was established which made it possible to develop more complex societies. However, it is the "copy-revolution" of the printing press that enables human stigmergy to be used at full scale throughout society. The reduced cost of making a book allowed for a much more flexible reuse and sharing of existing 
knowledge across wider geographical distances. All types of written knowledge could easily be copied and made accessible to many more readers. Human stigmergy evolved into new forms. Frozen stigmergic problem solving describes how existing solutions in book format were copied and reused at an unprecedented scale. The mass production of identical copies made it possible to spread the same message to everyone across large geographical areas. This led to a radical increase in available knowledge; people began to learn faster from each other because knowledge sharing was amplified. In addition, fluid stigmergic problem solving describes how knowledge products were not only copied, but they were improved through new book editions and translated, which further spurred collective knowledge advancement.

Chapter 9 argues that the origins of collaborative problem solving can be traced back to mutual collaboration, which built on the evolution of more advanced forms of gestural communication. Elaborative collaborative problem solving builds on this type of collaboration and requires proximate mutual interaction and sympathy between the collaborators. In contrast, rule-governed collaborative problem solving centers on an idea of fairness and requires that collaborators adhere to specific rules or norms in their collaboration. At least two collaborative cultures were key in the evolvement of this type of collaboration. First, stone tool learning required deliberate practice and the presence of a community of learners with norms. Explicit teaching and individual training built on purposeful activities that were considered valuable, a collaborative culture which over time made it possible to refine stone tools across generations. Second, it is likely that hunter-gatherer groups were important in the development of ideas on equal participation, building on reciprocity and norms that emphasized equal sharing of food. Calculated reciprocity represents a significant move away from the dominance of a few individuals in groups. Equal sharing of food required increased control of emotions and the establishment of norms that kept free riders out. A fair sharing of spoils also permitted role differentiation in groups because not everyone had to participate in the hunt in order to get food.

\section{I.5.4 Step 4: Design of CI}

The second part of the book examines in more detail how CI can be successful in the scientific and political domain. The analysis covers three interrelated dimensions: intelligent engagement (Chapter Io), intelligent contributions (Chapter II), and intelligent evaluations (Chapter I2). 
On one hand, CI points to the emergence of Open Science, an umbrella term that describes a movement that aims to make scientific research accessible to everyone in society. This involves both citizen participation in scientific processes and the increased open sharing of scientific publications. On the other hand, CI orientates itself towards Open Democracy, which is a term used to describe how the Internet and digital technology can strengthen democratic participation and put ordinary citizens at the center of political systems in new ways.

Chapter Io analyzes the relationship between citizen participation and citizen expertise, particularly in the political domain. New types of intelligent citizen engagement are emerging, such as mass deliberation, mass voting and social media activism. Mass deliberation describes the evolvement of new democratic institutions that aim to recruit citizens in direct participation. Two examples, the Citizens' Council in Ostbelgien and the online ideation platform Better Reykjavík, are part of this new trend. Mass voting is another type of citizen participation that has received increasing popularity because of the Internet. Technological platforms make it easy to enable everyone to vote, such as the Five Star Movement does with its party members in Italy. Social media activism has also become increasingly important. This involves both informal political debate and political activism, which the social movement My Stealthy Freedom exemplifies. All these CI projects build on different conceptions of participatory diversity. In addition, transparent collective work is important in promoting intelligent engagement between large groups, both in scientific work like crowd peer review or political processes like the Icelandic constitutional experiment. However, there is a concern about the threats to democracy that dysfunctional engagement presents, such as fake news and echo chambers in social media.

Chapter I I address how contributions are combined in different ways when designing CI. One approach utilizes many different perspectives in the same work, like in collective work on the same Wikipedia article. Multidisciplinary innovation teams also include a diversity of perspectives in creative problem solving. Second, contributions can be combined under the assumption that the golden middle way is the best solution. One example is the identification of a quantitative middle point, such as an average, that provides the most accurate solution if contributions are diverse. Another strategy is to find the middle way by developing a balanced representation of all sides, as in collective argument mapping. In addition, the middle way can identify commonalities, like the online environment vTaiwan that lets the crowd find consensual statements in 
political conflicts. A third approach scales up the number of contributions in the search for an unexpected solution. Many breakthrough ideas happen at the outskirts of a field. Online innovation contests aim to bring in creative outsiders or unknown others by inviting anyone to join. Furthermore, most of the contributions in CI projects build on a modularization strategy that splits a complex challenge into many smaller subtasks.

Chapter I 2 describes different types of intelligent evaluation. At all group levels, most CI practices are reliant on some degree of explicit evaluation of the collective work. Digital technology also makes it possible to design metacommunicative feedback loops in most group work and organizational work. While some systems build on shared coordination, others let coordinators regulate the collective work. In the political system, intelligent evaluations are at the core of any well-functioning democratic system, from the nomothetai in ancient Athens to the Citizen Assembly in Ireland today. These new institutions strengthen citizen metadiscourses about important societal issues. A strong knowledge commons is also an important basic condition for this type of critical discourse. In general, digitized evaluations are becoming more common in society, exemplified by online reputation systems that rate a person's trustworthiness, not only on business sites, but also in social media. However, there is increasing concern about the negative consequences of evaluating persons in the emerging reputation society.

Chapter I 3 describes COVID-I9 as a wicked problem and shows how different CI mechanisms have been used to cope with the pandemic. The first CI mechanism is the transparent flow of information during the pandemic. Knowledge is being shared at a rapid pace in the global online setting. Most of the big news sites provide citizens with updated statistics on the spread of the virus. Another example is the governmental "test and trace" strategy that aims to maximize information about the spread of the virus at all times. A second CI mechanism is citizen responsibility. Citizens in all countries have faced the challenge of complying with behavioral rules enforced by the government. Rules on social distancing and voluntary quarantines depend on citizen cooperation. Here, New Zealand stands out as one of the most successful countries. Third, collective learning at a system level has been important in dealing with the pandemic. One example is South Korea, which learned a lot from the Middle East Virus (MERS) in 2015 a couple of years before the COVID-19 outbreak. Their learning from the past failures in coping with that outbreak made them much better prepared than other countries. 
Chapter I4 explores what motivates individuals to contribute to $\mathrm{CI}$ projects. If we think $\mathrm{CI}$ can benefit society, we need to understand what motivates individuals to engage in collective problem solving. However, both the complexity of the tasks and the required skill levels varies a lot. It ranges from innovation contests that often look for individuals with specific formal qualifications to citizen science project that require simple image detection skills. In a historical perspective, we have more spare time than ever before and many CI projects depend on this extra "time resource", but the competition with social media and other entertainment services is fierce. The chapter examines a wide range of motivational factors, such as being immersed, being recognized, being part of a community, learning as motivation, economic motivation and making societal contributions. Statements from top solvers in online innovation contests comprise an important part of the content in this chapter.

Chapter 15 concludes by describing two radically different future visions of the intelligent society. On the one hand, instrumentarian intelligence assumes that algorithms tracking human behavior can predict human behavior more accurately than ever before. In Western countries, this intelligence manifests itself in a new surveillance capitalism, with companies like Google and Facebook constantly searching for behavioral surplus in both online and offline settings. In the political domain, instrumentarian intelligence seeks a reputation state built on a neobehavioristic governing model. The most prominent example is the nationwide social credit system in China that makes it possible to grade citizens on different behavioral indicators.

On the other hand, civic intelligence highlights a use of technology still controlled by the community and citizens, in contrast to the dehumanizing aspects of instrumentarian intelligence. While machine intelligence also craves for informational diversity in its hunt for behavioral surplus, civic intelligence seeks a broader diversity that includes not only information, but also multicultural, cognitive, biological and participatory diversity. The "fuel" of CI is people who are different from each other, with different interests and unique perspectives. Civic intelligence also builds on a strong knowledge commons and an open, shared collective memory. It does not hide information to produce the best predictions but promotes complete transparency and individual empowerment. In contrast to instrumentarian intelligence, CI still enables human-to-human intelligence, and not the algorithms, to be at the core of human collective problem solving. 